\title{
Scanning-Beam Microwave Landing System-Multipath-Errors and Antenna-Design Philosophy
}

\author{
ALFRED R. LOPEZ, SENIOR MEMBER, IEEE
}

\begin{abstract}
Multipath reception can cause guidance-angle errors in a microwave landing system (MLS). The antenna radiation-control problem for a scanning-beam MLS is defined and analyzed. "Centerline emphasis" is presented as a helpful design philosophy for the ground antennas. It is shown that the maximum guidance-angle error is proportional to 1) the amplitude of the indirect signal, 2) the antenna beamwidth, and 3 ) the time derivative of the indirect signal as the direct beam-peak scans by the receiver. This result is used in developing a rationale for the selection of the antenna beamwidth and sidelobe level (aperture size and excitation) for the azimuth and elevation guidance functions.
\end{abstract}

\section{INTRODUCTION}

$\mathbf{A}$ SCANNING-BEAM microwave landing system (MLS) is being developed by the FAA which is intended to replace the existing instrument landing system (ILS). One key feature of a microwave system is its capability of reducing multipath errors by utilization of narrow-beam antennas. The MLS has the capability for aircraft guidance down to touchdown and through rollout, even at sites with obstacles which cause reflected signals that are stronger than the direct signal.

The MLS multipath-error and antenna-design problem is an intriguing one, and one that has received a great deal of attention. Much work has been done in developing an understanding of the basic multipath phenomenon [1], [2] and how it affects system performance in terms of bias, path following, and noise-like errors. This, however, is not the main subject of this paper. Here the goal is to develop some principles for controlling the radiation from the ground transmitters so that the error caused by a reflecting obstacle is less than a specified value for a given obstacle reflection factor.

Presented in this paper are 1) a philosophy of design which proposes that a helpful approach for the design of the ground antennas is to emphasize radiation along the runway centerline, and 2) a basic relationship between the multipath error and the antenna beamwidth and sidelobe level.

This paper presents basic reference information for the design engineer whose task it is to specify the aperture size and excitation that will ensure that the multipath error is less than a specified value. Two design examples are given, one for the azimuth (AZ) and the other for the elevation (EL) guidance function. The objective for each of these examples is to highlight the basic design trade-offs. Although not

Manuscript received January 31, 1976; revised June 8, 1976. The basic multipath problem was defined and analyzed under contract to the FAA during the first two phases of the MLS development program [1].

The author is with the Wheeler Laboratory, Hazeltine Corporation, Greenlawn, NY 11740.

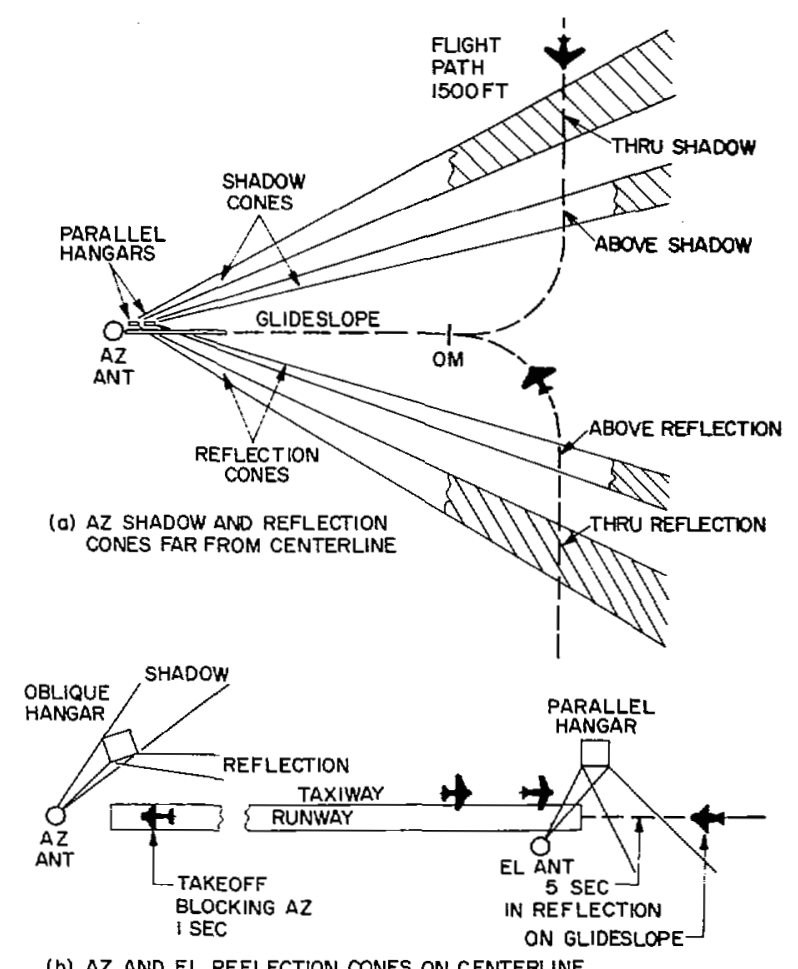

(b) AZ AND EL REFLECTION CONES ON CENTERLINE

Fig. 1. Airport runway environment.

definitive, an attempt is made to use reflection factors and error tolerances which are consistent with actual airport obstacles and system requirements.

\section{PROBLEM DEFINITION}

The time reference scanning beam (TRSB) MLS [2] provides angle guidance to an aircraft by scanning a fan beam back and forth across an angular region at a prescribed rate. The angle information is decoded by measuring the time between the peaks of a pair of scans at the aircraft receiver. A basic MLS problem is to achieve the required accuracy in the presence of airport obstacles which can cause coding errors.

Fig. 1 shows a plan view of an airport runway environment showing the antennas for the azimuth and elevation angleguidance functions [1]. Two regions on the airport can be described which have special significance for a landing system. The first, shown in Fig. 1(a), is the area away from the runway centerline, where a large hangar or other building may cause a cone of deep shadow and a cone of strong reflection. These cones may or may not be high enough to intersect the flight path. The deep shadow is a problem inherent in any micro- 


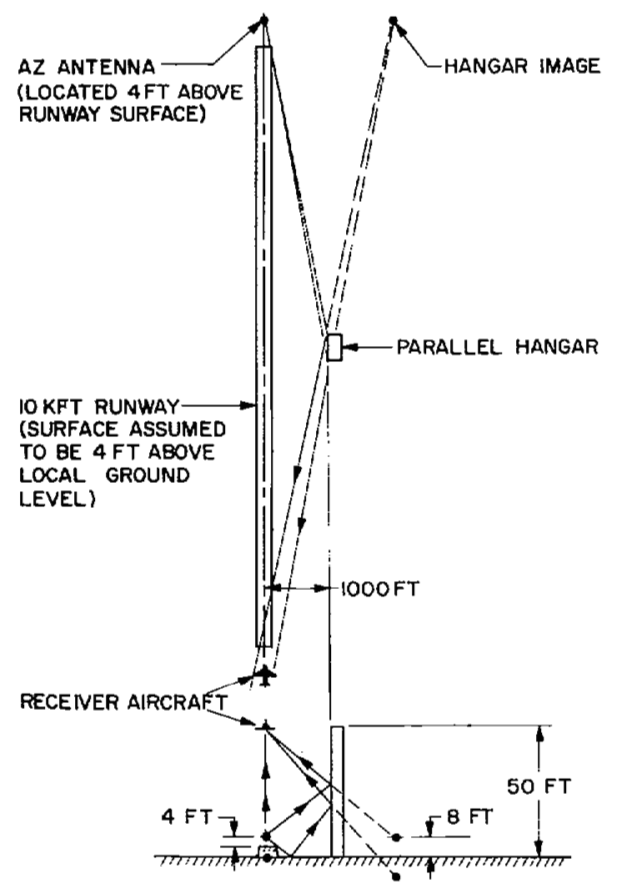

Fig. 2. Possible situation of indirect signal that is $6 \mathrm{~dB}$ stronger than direct signal.

wave system using a low antenna for wide-angle coverage. This is an acquisition and validation problem, beyond the scope of this paper. The strong reflection is discussed below for the more critical case of an aircraft on centerline approach.

The second region, shown in Fig. 1(b), is the area near the runway centerline, which is free of any large fixed obstacle capable of causing a deep shadow or a strong reflection. Here, one concern is any other aircraft intervening between the aximuth transmitter and the approaching aircraft. Such an aircraft may cause a momentary partial blocking on takeoff over the antenna, or more persistent partial blocking while on the runway. Neither case is a deep shadow. Thus shadowing is not a major problem for aircraft on centerline approach.

A principal problem for aircraft on centerline approach is the errors caused by the multipath reception of the direct signal and the indirect signals reflected by hangars and by the ground. In a reflection cone, the reflected signal can be stronger than the direct signal. Fig. 2 shows a typical situation of a runway with the azimuth antenna close to the ground and near the stop-end of the runway, the aircraft near threshold, and a parallel hangar. (The antenna is located near the ground in order to provide adequate clearance for aircraft and to suppress the elevation lobing caused by the ground reflection.) The runway surface is shown $4 \mathrm{ft}$ above the local ground. For this case, since both the antenna and receiver are near the ground, the sum of the direct signal and its ground reflection (voltage ratio) is proportional to the antenna height ( $4 \mathrm{ft}$ ), and the sum of the indirect signal and its ground reflection is proportional to the height of the hangar image $(8 \mathrm{ft})$. The result is an indirect signal that may be $6 \mathrm{~dB}$ stronger than the direct signal. This result is modified by the relative antenna gain in the directions of the aircraft and the hangar, the path-length ratio, and the reflection coefficient for the hangar [1]. All of these factors can be near unity. The net result is that an indirect signal can be stronger than the direct signal. This is also true for the elevation function.

The antenna design problem is to provide angle coding in space while also providing the necessary radiation control (spatial filtering) to reduce multipath errors to tolerable limits.

\section{CENTERLINE EMPHASIS}

It is believed that an aircraft on centerline should have the highest priority and it is apparent that off-centerline obstacles present a serious threat. This leads to the "centerline emphasis" concept in the design of a microwave landing system [1], [2] . That is, to emphasize radiation along the centerline and to suppress, as much as possible, radiation in the direction of reflecting obstacles while providing the required angular coverage. This design philosophy is applied to the examples given below for the $\mathrm{AZ}$ and $\mathrm{EL}$ antennas. Centerline emphasis is found to be especially helpful for the elevation function.

(The concept of centerline emphasis is not unique to MLS. Existing ILS localizers (azimuth quidance) at some difficult sites, use two array antennas or a large-aperture $\mathrm{V}$-ring array antenna to provide a coarse guidance signal at wide angles, and an accurate guidance signal on centerline [6] .)

\section{RELATIONSHIP OF MULTIPATH ERROR TO ANTENNA BEAMWIDTH AND SIDELOBES}

It is possible to relate the error caused by an indirect signal to the antenna beamwidth and sidelobe level. The parameters which must be related are

1) maximum angle-coding error $\delta$,

2) indirect/direct signal voltage ratio $\rho$,

3) aperture/wavelength ratio, $D / \lambda$,

4) antenna beamwidth (nominally $\lambda / D$ ),

5) antenna sidelobe level.

The angle-coding error is defined as the displacement of the beam-peak in time at a point in space cuased by an indirect signal. Fig. 3(a) shows the general case of an electronic scanning-beam antenna, a receiver, and a reflector. The direct signal in time at a point in space is a replica of the transmitted antenna array factor, $F(u)$; the location of the beam peak in time depends on the location of the receiver in space. Fig 3(b) shows the received direct and indirect signal components as the scan angle yaries with time ( $u=K t, K=$ scan rate). The sum of the two components has a main beam whose peak is displaced; the maximum amount of displacement in time $(\Delta t)$ or angle error $(\delta=(\lambda / D) K \Delta t)$ can be determined for the case of $\delta \ll$ $\lambda / D$. For this case, the sum of the direct and indirect signals is given by

$$
\Sigma F=F(u)+\rho F\left(u-u_{s}\right) .
$$

The indirect/direct signal ratio $\rho$ is defined as the product of four time-invariant factors: 1) array-element gain; 2) pathlength ratio, 3) elevation lobing, and 4) a reflection factor 


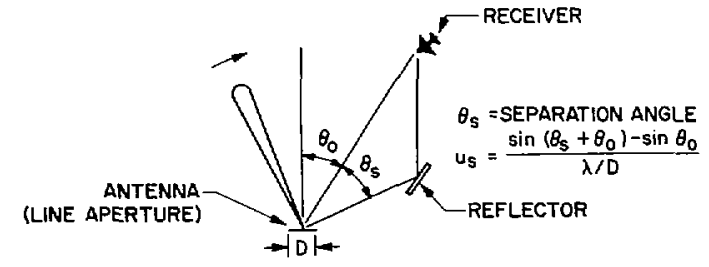

(a) GEOMETRY

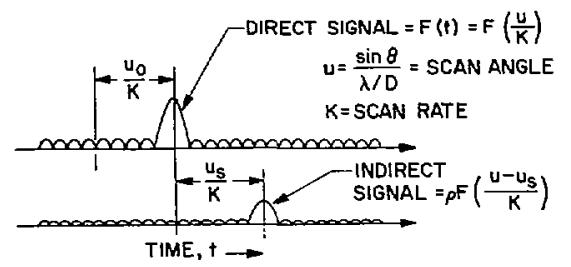

(b) RECEIVED SIGNAL COMPONENTS

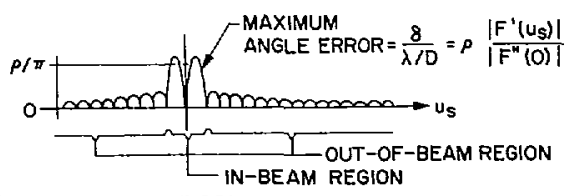

(c) ANGLE ERROR PATTERN

Fig. 3. Multipath angle-error pattern.

which depends on the reflector size, flatness, and surface reflectivity.

The leading terms of the series expansion of $\Sigma F$ about the peak of the direct signal are

$$
\begin{aligned}
\Sigma F= & F(0)+F^{\prime \prime}(0) \frac{u^{2}}{2} \\
& +\rho F\left(u_{s}\right)+\rho F^{\prime}\left(u_{s}\right) u+\rho F^{\prime \prime}\left(u_{s}\right) \frac{u^{2}}{2} .
\end{aligned}
$$

Differentiating and equating to zero results in

$$
\left(F^{\prime \prime}(0)+\rho F^{\prime \prime}\left(u_{s}\right)\right) u_{\delta}+\rho F^{\prime}\left(u_{s}\right)=0
$$

where

$$
u_{\delta}=\frac{\delta}{\lambda / D}
$$

By solving this equation for $u_{\delta}$, the relationship between the maximum angle error (or maximum beam-peak displacement in time, $\left.u_{\delta} / K\right)$ and the separation angle is obtained for $\rho\left|F^{\prime \prime}\left(u_{s}\right)\right| \ll\left|F^{\prime \prime}(0)\right|$ (this condition requires small $\rho$ or low sidelobes). This relationship is defined as the angle-error pattern and is given by

$$
\delta=\frac{\rho}{\pi} \frac{\lambda}{D} k\left(u_{s}\right)
$$

where

$$
k\left(u_{s}\right)=\pi\left|\frac{F^{\prime}\left(u_{s}\right)}{F^{\prime \prime}(0)}\right|=\text { multipath error factor. }
$$

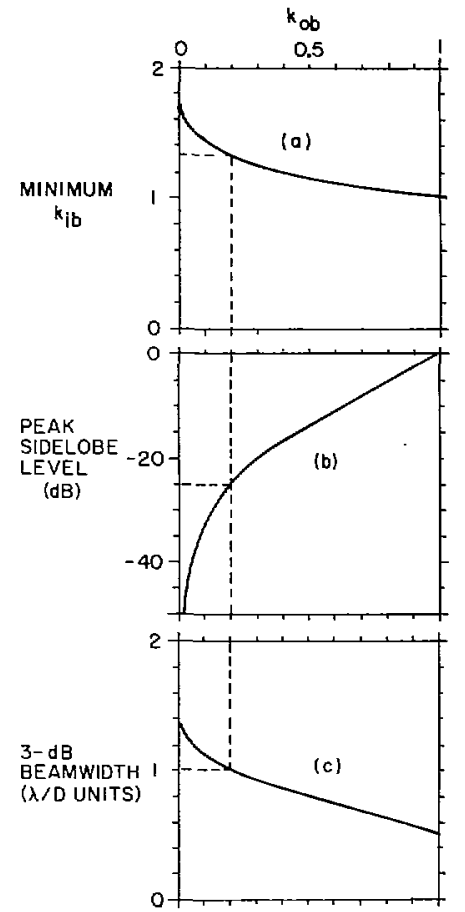

Fig. 4. Relationship of multipath error to antenna beamwidth and sidelobe level.

Thus the maximum angle error is proportional to 1) the indirect/direct signal ratio $\rho, 2)$ the nominal or aperture beamwidth $\lambda D$, and 3$)$ the derivative of the indirect signal $F^{\prime \prime}\left(u_{s}\right)$.

As shown in Fig. 3(c), the angle-error pattern has two regions which have special significance: the in-beam and outof-beam regions.

Definitions:

$k_{i b}$ is the maximum value of first lobe of $k\left(u_{s}\right)$ is the maximum in-beam error factor,

$k_{o b}$ is the upper bound for all sidelobes of $k\left(u_{s}\right)$ is the maximum out-of-beam error factor.

There is a basic trade-off between $k_{o b}$ and the minimum $k_{i b}$. Fig. 4(a) shows this trade-off, a decrease of $k_{o b}$ results in an increase in the smallest possible value for $k_{i b}$. It is also noted that

$\min k_{i b} \geqslant 1 \geqslant k_{\mathrm{ob}}$.

The curves of Fig. 4 are computed for the Chebyshev patterns (minimum beamwidth for a given sidelobe level) which have been modified to provide near minimum beamwidth and near the minimum value for $k_{i b}$ for a given $k_{o b}$. The Chebyshev patterns have constant sidelobe levels; the corresponding error-factor patterns, as shown in Fig. 5, have tapering sidelobes. The Chebyshev excitations are modified by increasing the characteristic impulse excitations at each edge of the aperture [3] to provide error-factor patterns with the first and far sidelobes at equal levels. For a given $k_{o b}$, the modified Chebyshev pattern has a narrower beamwidth and smaller $k_{i b}$ than the unmodified Chebyshev pattern. The 
CHEBYSHEV PATTERN FUNCTION, $25 \mathrm{~dB}$ SIDELOBES

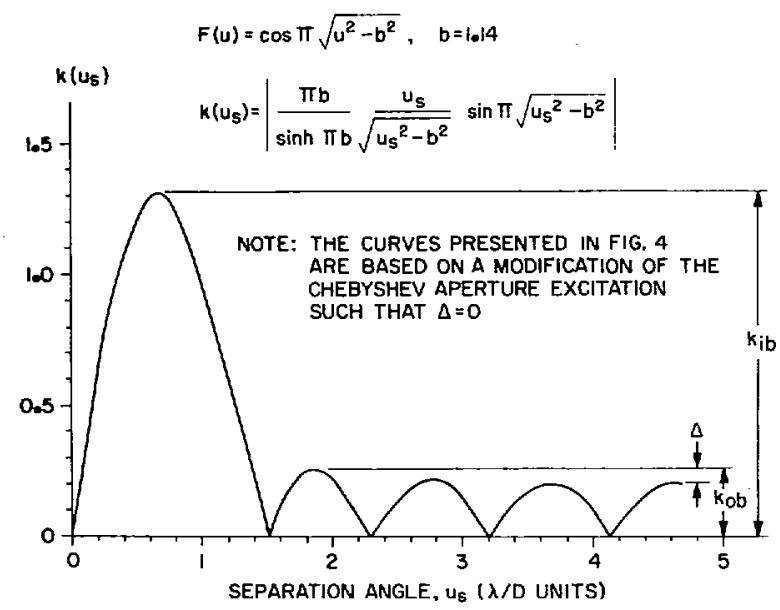

Fig. 5. Chebyshev multipath-error-factor pattern.

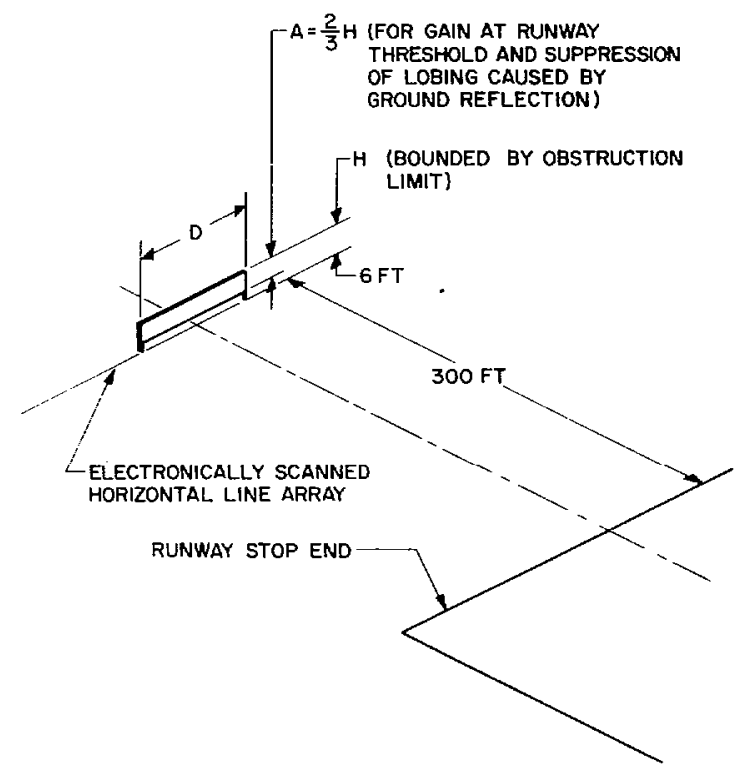

Fig. 6. Azimuth antenna.

modified Chebyshev patterns are very close to optimum with respect to minimum beamwidth and minimum-maximum in-beam error for a given $\rho$ and a given maximum out-of-beam error.

Fig. 4 is the principal result of this paper. It shows the relationship of $k_{o b}$, minimum $k_{i b}$, sidelobe level, and 3-dB beamwidth for the modified Chebyshev functions. The curves of Fig. 4 are typical and show trends. They can be used to specify the minimum aperture size and the excitation that will ensure that the multipath error is less than a specified value for a given obstacle reflection factor. Two examples are presented in the following sections.

\section{AZIMUTH-GUIDANCE ANTENNA DESIGN}

The basic azimuth-guidance antenna is an electronically scanned horizontal line array as shown in Fig. 6. The array factor, which is scanned in time to provide angle coding, can

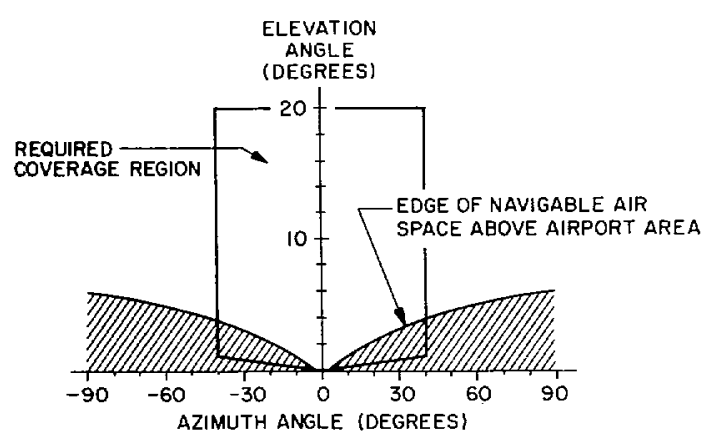

Fig. 7. Horizon for possible airport obstacles as viewed from azimuth antenna.

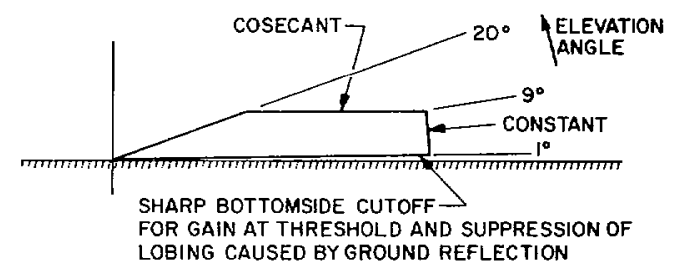

Fig. 8. Idealized vertical-plane pattern for azimuth antenna.

be controlled to provide spatial filtering. The radiating-element pattern provides another (direct) spatial-filtering capability.

The azimuth antenna radiates a fan beam that is wide in elevation and narrow in azimuth, and is scanned in azimuth. The design problems for this antenna include the control of radiation in both the elevation and the azimuth planes.

Fig. 7 shows a view from the $\mathrm{AZ}$ antenna at the stop-end of an airport runway. The horizon shown in the figure is the lower edge of the navigable air space above the airport area as presently defined for ILS runways [4]. Almost all large airport obstacles present profiles which lie in the shaded area. A good design objective for the azimuth antenna is to suppress, as much as permissible, the radiation in directions within the shaded area.

The radiation-control problem in the vertical plane is to suppress the lobing caused by the ground reflection and to provide the required vertical plane coverage. Fig. 8 shows an idealized pattern for the vertical fan beam. The principles and techniques for controlling ground lobing and for shaping elevation patterns have been described [5], [7]. The proper vertical excitation of the upper $2 / 3$ of the available aperture (see Fig. 6) will provide gain at the runway threshold and will suppress elevation lobing.

A challenging design problem is determining the horizontal aperture size and excitation for a specified tolerance of multipath error. Fig. 9 shows the pattern factors for the horizontal array. The radiated pattern is the product of the element factor, which is fixed in angle, and the array factor, which is scanned. A typical element pattern and an idealized element pattern are shown. The idealized pattern radiates only in the required coverage region and emphasizes radiation along the runway centerline. If achievable in practice, a pattern of this type would significantly enhance the overall performance of the azimuth antenna. The element pattern is a spatial filter which can suppress radiation in the direction of reflecting 

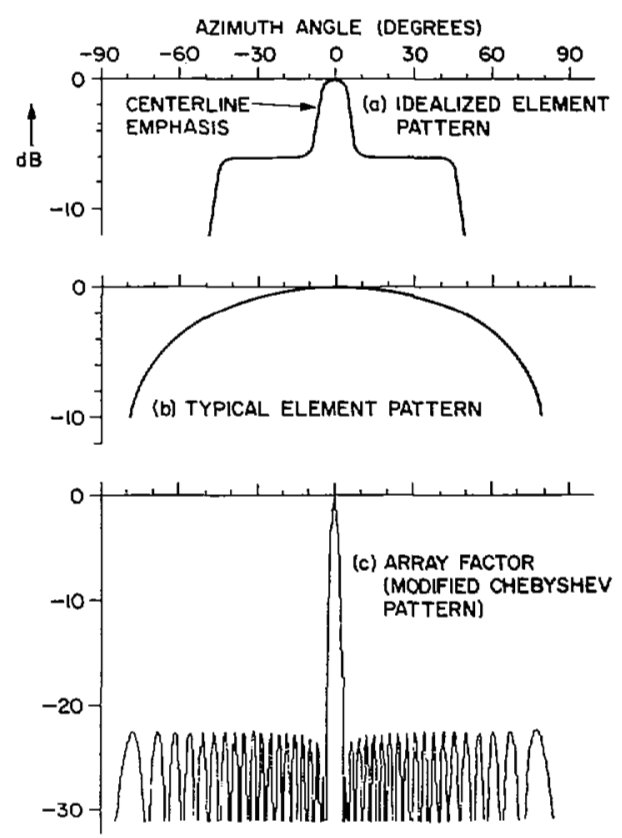

Fig. 9. Element and array-factor patterns for azimuth antenna.

obstacles. In the discussion below, however, the element pattern factor is assumed to be uniform (omnidirectional), more like Fig. 9(b).

As noted in Section II, the indirect signal may be $6 \mathrm{~dB}$ stronger than the direct signal and, conceivably, could create a false course. This is an acquisition and validation problem that has alternative solutions. This subject, however, is beyond the scope of this paper. The concern here is the error caused by sidelobe radiation reflected off an obstacle and arriving at the receiver as the peak of the direct beam scans by the receiver.

The results given in Fig. 4 can be used to determine the minimum aperture size $(D / \lambda)$ and antenna sidelobe level that will ensure that the multipath error is less than a specified value, for the most critical case of the receiver on centerline approach. For this case, all hangars are out-of-beam, therefore the curve of Fig. 4(b) can be used to determine the desired relationship. The following example is given.

Problem: Determine the minimum horizontal aperture size so that the multipath angle error $\delta$ is less than $0.06^{\circ}$ (corresponds to a 10 -ft error at the threshold of a $10 \mathrm{kft}$ runway) for the following conditions:

1) receiver on centerline approach,

2) $-25 \mathrm{~dB}$ antenna sidelobes,

3) omnielement pattern in horizontal plane,

4) reflecting obstacle may be located at any angle greater than $4^{\circ}$ off the runway centerline,

5) indirect/direct signal ratio, $\rho=0.5,1$, or 2 .

The answer is determined immediately from the curve of Fig. $4(\mathrm{~b})$ by entering at the $-25 \mathrm{~dB}$ sidelobe-level point and reading

$$
k_{o b}=0.20 \text {. }
$$

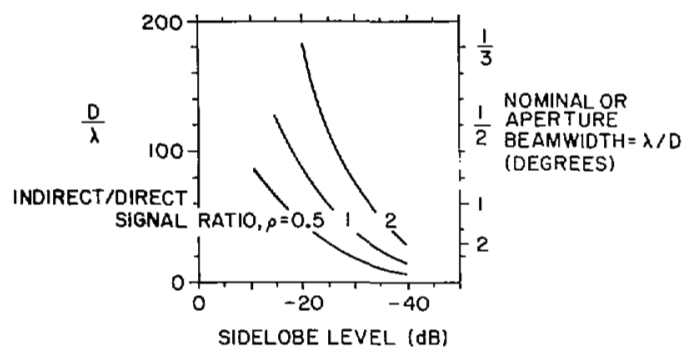

Fig. 10. Aperture size versus sidelobe level for specified out-of-beam error tolerance, $\delta=0.06^{\circ}$.

Therefore, from (1)

$$
\begin{aligned}
\frac{D}{\lambda} & =\frac{1}{\delta} \frac{\rho}{\pi} 0.2 \\
& =30,60,120 ; \quad \text { for } \rho=0.5,1,2 .
\end{aligned}
$$

It is more instructive to replot the curve of Fig. $4(\mathrm{~b})$ in terms of $D / \lambda$ versus sidelobe level for $\delta=0.06^{\circ}$, for the three values of $\rho$. This relationship is given in Fig. 10 which shows the basic design tradeoff for the AZ antenna. If the obstacle is such that $\rho=2$ (which is not uncommon, as discussed in Section II) the antenna designer may select from one of many possible designs. A few of these are 1$)-20 \mathrm{~dB}$ sidelobes and $D / \lambda=182,2)-25 \mathrm{~dB}$ sidelobes and $D / \lambda=120$, or 3) -30 $\mathrm{dB}$ sidelobes and $D / \lambda=73$. Recognizing the difficulty in achieving $-30 \mathrm{~dB}$ sidelobes in practice, the antenna designer may have to select a $D / \lambda$ of about 120 for a practical design, to satisfy the $0.06^{\circ}$ error specification. Weaker indirect signals would, of course, require less aperture size.

This example points out that one of the most important design considerations for the $A Z$ antenna is the tradeoff of aperture size against the difficulty of maintaining sidelobes below a certain level. This consideration is important in the overall evaluation of performance and cost.

\section{ELEVATION-GUIDANCE ANTENNA DESIGN}

The basic elevation-guidance antenna is an electronically scanned vertical line array located as shown in Fig. 11. This antenna radiates a fan beam that is wide in azimuth and narrow in elevation, and is scanned in elevation. The design problems for this antenna include the control of radiation in both the azimuth and elevation planes.

Fig. 12 shows a view from the elevation antenna site. As in Fig. 7, the horizon shown in the figure represents the lower edge of the navigable air space above the airport area. For the elevation guidance case, hangars in the cross-hatched region can cause large in-beam errors which can only be reduced by radiation control in the horizontal plane and by motion averaging. (When the angle of arrival between the direct and indirect signals is large, a short-wavelength standing-wave pattern exists along the flight path which causes a rapid cyclic variation of the multipath error. This effect can be utilized to reduce the error by averaging several angle measurements.) 


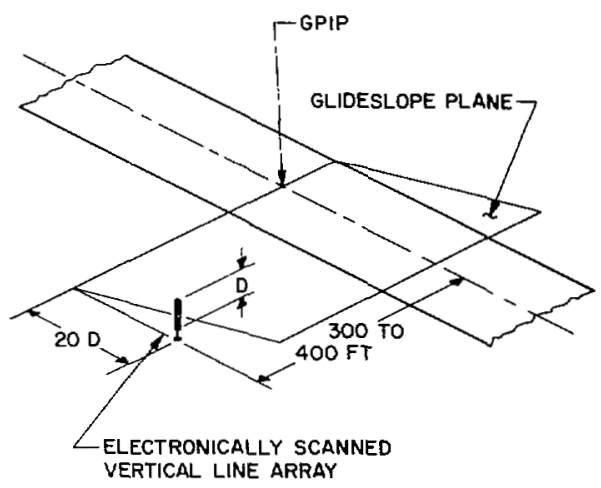

Fig. 11. Elevation antenna.

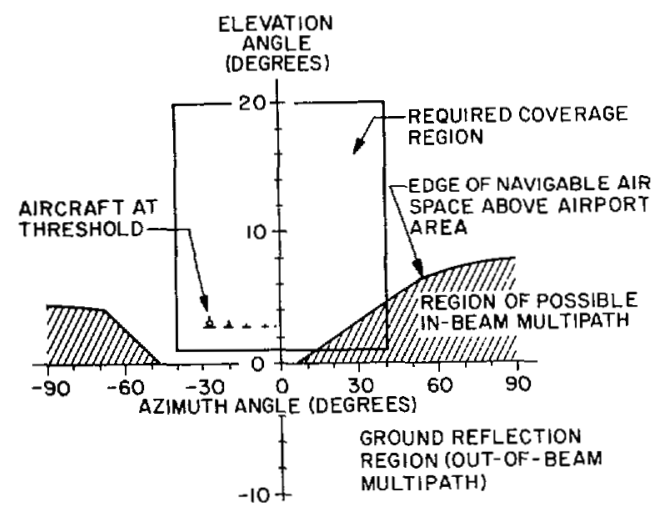

Fig. 12. Horizon for possible airport obstacles as viewed from elevation antenna.

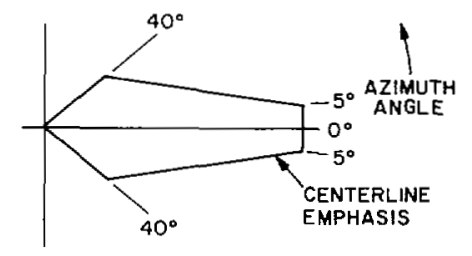

Fig. 13. Idealized horizontal-plane pattern for elevation antenna.

Fig. 13 shows an idealized horizontal-plane pattern shaped to provide the required coverage and to reduce the in-beam errors caused by hangars. With this centerline-emphasis pattern a unity reflection factor for hangars is reduced to about $1 / 2$. This factor is further reduced, typically, to $1 / 4$ by the pathlength-ratio factor. For the elevation guidance function, centerline emphasis is especially helpful for the reduction of in-beam errors.

Fig. 14 shows an idealized element pattern for the vertical line array which can be approximated in practice. A pattern of this type would allow for a higher-sidelobe array factor or, possibly, a smaller aperture size for the same sidelobe level. In the discussion below, however, it is assumed that the element pattern is uniform.

Fig. 4 can be used to determine the vertical aperture size and the required motion-averaging factor that will ensure that the in-beam and out-of-beam errors are less than a specified value. The following example is given.

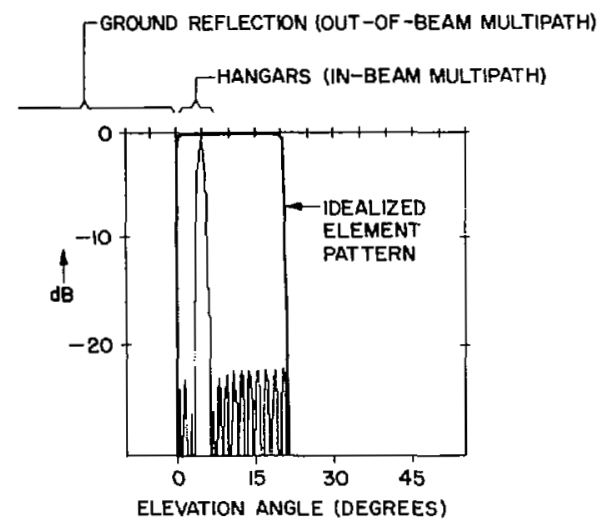

Fig. 14. Idealized element pattern for elevation antenna.

Problem: Determine the minimum vertical aperture size so that the out-of-beam angle error $\delta_{o b}$ is less than $0.06^{\circ}$ (corresponds to a $1-\mathrm{ft}$ error at the runway threshold) and determine the amount of motion-averaging error reduction required so that the in-beam error $\delta_{i b}$ is also less than $0.06^{\circ}$ for the following conditions:

1) receiver on centerline approach,

2) $-25 \mathrm{~dB}$ sidelobe level,

3) omnielement pattern in vertical plane,

4) $\rho_{i b}=1 / 4$ (hangar reflection and centerline emphasis),

5) $\rho_{o b}=1$ (ground reflection).

The aperture size is determined by entering the curve of Fig. 4(b) at the $-25 \mathrm{~dB}$ level:

$$
k_{o b}=0.20 \text {. }
$$

Therefore, from (1)

$$
\frac{D}{\lambda}=60
$$

It is noted that the same trade-off of aperture size and sidelobe level, described for the $\mathrm{AZ}$ example, is applicable here.

The required amount of motion-averaging error reduction is determined by first reading from the curve of Fig. 4(a)

$$
k_{i b}=1.32 \text {. }
$$

Therefore, from (1)

$$
\delta_{i b}=\frac{\rho}{\pi} \frac{\lambda}{D} 1.32=0.10^{\circ} .
$$

Thus a motion-averaging error-reduction factor of 0.6 is required, to ensure that the in-beam error is less than $0.06^{\circ}$.

This example points out that for the EL antenna, the reduction of in-beam error is a difficult problem. Typically, both centerline emphasis and motion averaging are required to reduce this error. If these error-reducing methods are not utilized, larger apertures would be required to ensure that the in-beam error does not exceed the tolerance. 


\section{CONCLUSION}

A basic problem for MLS is the errors caused by indirect signals reflected by hangars and by the ground. The ground antenna design problem is to reduce these multipath errors by spatial filtering while providing the required angle coding in space.

"Centerline emphasis" is an important design concept for MLS. The principle is to emphasize radiation along the runway centerline and to suppress radiation, as much as permissible in the directions of reflecting obstacles. This approach is found to be especially helpful for the elevation antenna.

A basic relationship is shown to exist between the multipath error and the signal radiated by the ground antenna, which is that the maximum angle error is proportional to 1) the amplitude of the indirect signal, 2) the antenna beamwidth, and 3) the time derivative of the indirect signal as the direct beam-peak scans by the receiver. Based on this result a set of curves are computed for a particular pattern shape (modified Chebyshev) which relate multipath error to antenna beamwidth and sidelobe level. These curves are typical and show trends. They are helpful in determining the minimum aperture size and the excitation that will ensure that the multipath error is less than a specified value.

In the design examples presented, it is pointed out that a very important consideration with regard to cost versus performance, is the trade-off of aperture size against the difficulty of maintaining sidelobes below a certain level. This is true for both the azimuth and elevation antennas.

\section{ACKNOWLEDGMENT}

The stimulus for the work described in this paper was acquired during the writer's participation in the MLS technique selection activity in the fall of 1974. During this period the FAA MLS Program Office brought together a group of experts and specialists in many disciplines, from government and industry, from the United States and other countries, to participate in the difficult process of selecting between two competing MLS techniques, scanning beam and Doppler. One of the most important by-products of this activity was the overall increase in the level of understanding of the basic capabilities and limitations of a microwave landing system.

The writer would like to acknowledge the contributions, and the help, support, and encouragement of his many associates at Hazeltine Corporation. In particular, he is most appreciative of the teaching and patience of Dr. Harold A. Wheeler.

\section{REFERENCES}

[1] H. A. Wheeler and A. R. Lopez, "Multipath Effects in Doppler MLS," Multipath section of Hazeltine Report 10926, "FiveYear Microwave Landing System Development Program Plan," September 1972; Hazeltine Reprint H-222; October 1974.

[2] Federal Aviation Administration "Time Reference Scanning Beam MLS-Submission to the International Civil Aviation Organization," December 1975 (see p. 1-3.75 for a list of references on multipath).

[3] A. R. Lopez, "Line-Source Excitation for Maximum Aperture Efficiency with Given Sidelobe Level," IEEE Trans. Ant. Prop., Vol. AP-19, pp. 530-532, July 1971.

[4] Federal Aviation Administration, "Objects Affecting Navigable Airspace," Federal Aviation Regulations, Part 77, May 1965.

[5] R. J. Giannini, J. H. Gutman, and P. W. Hannan, "A Cylindrical Phased-Array Antenna for ATC Interrogation," Microwave Journal, October 1973.

[6] M. Kayton and W. R. Fried, Avionics Navigation Systems. New York: Wiley, 1969 , p. 536.

[7] R. F. Frazita, "Aperture Location for Minimum Ground Reflection," Hazeltine D-R390S, January 1973. 